\title{
Factores de riesgo para infección por Pseudomonas aeruginosa multi-resistente en un hospital de alta complejidad
}

\author{
Ana Claudia Ossa-Giraldo, Lina María Echeverri-Toro, Zila Margarita Santos, \\ Mónica Giseth García, Yuli Agudelo, Faiver Ramírez y Sigifredo Ospina
}

\author{
Risk factors for multidrug-resistant Pseudomonas aeruginosa infection, \\ in a tertiary hospital in Colombia
}

Introduction: multi-resistant Pseudomonas aeruginosa (MR) is frequently associated with healthcare infections. Its epidemiology is complex and few studies help to understand it. A study about risk factors associated with this type of bacteria is needed. Objective: To determine risk factors associated with MR P. aeruginosa infection in hospitalized patients from the Hospital Universitario San Vicente Foundation-Medellin. Materials and Methods: case-control study to identify risk factors associated with infection by MR P. aeruginosa. Results: 140 patients were included, 70 in each group. Bivariate analysis found association with previous use of carbapenems (OR 3.12 - IC 1.21 to $8.03, \mathrm{p}=0.02$ ), aminoglycosides (OR $5.09-\mathrm{CI}: 1.38$ to $18,77, \mathrm{p}=0.01$ ) and days of stay prior to isolation of the organism (OR 1.03 - CI: $1.01-1.05, \mathrm{p}=0.01)$. In multivariate analysis MR P. aeruginosa infection was associated with hospital stay (OR 1.03 - IC 1.01 to 1.05), use of aminoglycosides (OR 1.30 to 19.28) and treatment with two or more antimicrobials in the last 30 days (OR 3.09 - CI: 1.26 to 7.58). The risk of developing infection was 3\% per day of hospital stay prior to isolation of the agent. Conclusion: Developing MR P. aeruginosa infection was associated with prior use of antimicrobials and prior hospital stay.

Key words: Pseudomonas aeruginosa, multidrug resistance, risk factors, Colombia.

Palabras clave: Pseudomonas aeruginosa, multi-resistencia, factores de riesgo, Colombia.

\section{Introducción}

$\mathrm{E}$ 1 aislamiento de bacterias resistentes a múltiples clases de antimicrobianos es una realidad del día a día en la práctica clínica. Dos de los factores que han favorecido el aumento de la frecuencia de infecciones por estos microorganismos son el uso indiscriminado de los antimicrobianos y la fácil diseminación de los mecanismos de resistencia entre las bacterias ${ }^{1-3}$.

Pseudomonas aeruginosa es causa frecuente de infecciones asociadas al cuidado de la salud, principalmente en pacientes inmunosuprimidos, quemados, personas con fibrosis quística y usuarios de unidades de cuidados intensivos (UCI); comúnmente presenta resistencia a varias clases de antibacterianos dificultando el tratamiento y asociándose a tasas mayores de mortalidad e incrementos en el costo de la atención hospitalaria ${ }^{4-6}$.

Según el estudio SENTRY (Antimicrobial Surveillance Program) en su reporte de los años 1997 y 2001, América Latina presentó niveles de resistencia antimicrobiana más altos que otras regiones evaluadas como Estados Unidos de América y Europa?

El Programa Europeo para la Vigilancia de Resistencia a Antimicrobianos (EARSS) publicó en 2007, tasas de resistencia a carbapenémicos mayores a $25 \%$ en seis países, teniendo Grecia la mayor tasa de resistencia notificada $(51 \%)^{8}$.

El panorama de la resistencia en Colombia también es preocupante; el Grupo para el Estudio de la Resistencia a Antibióticos de Medellín (GERMEN) reportó para el año 2011 tasas de resistencia de $P$. aeruginosa en UCI de $51,2 \%$ a aztreonam, $69,2 \%$ a ceftazidima, $72,7 \%$ a ciprofloxacina, $68,8 \%$ a imipenem, $70,4 \%$ a meropenem y $73,2 \%$ a gentamicina ${ }^{9}$, siendo este microorganismo el cuarto más aislado en las UCI y salas generales de los hospitales de la ciudad de Medellín, después de Escherichia coli, Staphylococcus aureus y Klebsiella pneumoniae.

Pseudomonas aeruginosa posee varios mecanismos de resistencia, tanto intrínsecos como adquiridos, que actúan independientemente o en conjunto, resultando en la expresión de resistencia frente a varias familias de antibacterianos como los $\beta$-lactámicos, aminoglucósidos, quinolonas y sulfonamidas ${ }^{10-12}$. Entre los mecanismos intrínsecos se encuentran la reducción de la permeabilidad que tiene la membrana plasmática a los antimicrobianos, sumado a la acción de las bombas de expulsión especialmente la bomba $\mathrm{MexAB-OprM}$ que actúa sobre una amplia gama de fármacos ${ }^{11,13}$; además, posee una
Universidad Cooperativa de Colombia, Facultad de Medicina. Medellín, Colombia (ACO). Institución Universitaria Colegio Mayor de Antioquia. Medellín, Colombia (ZMS, MGG). Hospital Universitario de San Vicente Fundación. Medellín, Colombia (LME, YA, SO). Universidad de Antioquia. Medellín, Colombia (FR).

Lugar de desarrollo de la investigación: Hospital Universitario de San Vicente Fundación.

Los autores declaran que no hay conflicto de interés alguno en el desarrollo de la investigación.

Fuente de financiamiento: Hospital Universitario de San Vicente

Fundación.

Recibido: 2 de abril de 2013 Aceptado: 22 de mayo de 2014

Correspondencia a: Ana Claudia Ossa Giraldo ana.ossa@campusucc.edu.co 
$\beta$-lactamasa cromosómica inducible tipo AmpC, que produce resistencia a piperacilina, aztreonam, ceftazidima y cefepima ${ }^{10,11}$. También posee $\beta$-lactamasas plasmídicas, carbapenemasas tipo metalo- $\beta$-lactamasas y una nucleotidil-transferasa y acetil-transferasa que actúan sobre los aminoglucósidos ${ }^{11}$. Otro mecanismo de resistencia de esta bacteria es la alteración de los sitios blanco de los antimicrobianos como ocurre con las fluoroquinolonas, en los cuales se han observado mutaciones sobre el gen gyr $A$ que codifica la subunidad A de la enzima ADN girasa, además de la alteración de las porinas, que impide la entrada del compuesto al microorganismo ${ }^{11}$; por ejemplo, la pérdida de la porina $O p r D$ por mutación, a partir de la cual aparece resistencia a imipenem y una disminución de la sensibilidad a meropenem ${ }^{10,11,13}$.

En esta bacteria no fermentadora, mecanismos de panresistencia como la producción de metalo-carbapenemasas (VIM-2, VIM-8) y serin-carbapenemasas (KPC-2) ya han sido descritos en los hospitales de este país ${ }^{14-16}$, limitando el tratamiento de los pacientes a antibacterianos tóxicos y que muestran bajas tasas de curación como son las polimixinas ${ }^{17}$. Las medidas enfocadas a intervenir los factores de riesgo asociados a la presencia de multi-resistencia han demostrado ser las más costo efectivas ${ }^{18-20}$.

El panorama mundial de resistencia bacteriana ha llevado a que se realicen mayores esfuerzos encaminados a la instauración de programas estrictos de control y vigilancia de infecciones, enfocados en disminuir la diseminación de estos microorganismos e impactar de forma positiva los desenlaces como morbilidad, mortalidad y estancia hospitalaria prolongada. Debido a esto es importante estudiar y determinar las fuentes, prevalencia y factores de riesgo de las infecciones causadas por estos microorganismos en el ambiente hospitalario de cada institución, para contribuir de manera importante al desarrollo de dichos programas. Atendiendo a estas necesidades, el objetivo de este estudio fue determinar los factores de riesgo asociados a infección por $P$. aeruginosa multiresistente (MR) en pacientes hospitalizados en el Hospital Universitario de San Vicente Fundación (HUSVF) de la ciudad de Medellín en Colombia.

\section{Métodos}

Se realizó un estudio analítico de casos y controles no pareado para determinar factores de riesgo asociados a infección por $P$. aeruginosa MR.

La población estuvo conformada por pacientes de cualquier edad y sexo, atendidos en el Hospital Universitario de San Vicente Fundación de Medellín, en el período comprendido entre 1 de enero de 2009 y 31 de diciembre de 2010, de quienes se aislara $P$. aeruginosa como causante de infección. A partir de esta población se seleccionó una muestra por conveniencia de 70 casos y 70 controles.

Se consideró "caso" a aquel paciente hospitalizado en el período de estudio con diagnóstico de infección por $P$. aeruginosa MR de acuerdo al perfil del antibiograma, el cual fue definido como resistencia a carbapenémicos o resistencia a dos o más familias de antibacterianos. Se consideró "control" a aquel paciente con diagnóstico de infección por $P$. aeruginosa sensible.

La identificación de los aislados y la determinación de las concentraciones inhibitorias mínimas (CIM) fueron realizadas por el equipo automatizado VITEK2 (bioMérieux Inc., USA). Las CIM fueron clasificadas de acuerdo con las directrices del Instituto de Estándares de Laboratorio Clínico (CLSI por sus siglas en inglés) de 2009.

Los datos se tomaron de la historia clínica del paciente. Las variables evaluadas fueron: presencia de co-morbilidades, tratamiento inmunosupresor o procedimiento quirúrgico durante la hospitalización, quemadura como causa de la hospitalización, muestra biológica en la que se aisló $P$. aeruginosa, estancia en la unidad de cuidados intensivos (UCI) al momento del aislamiento u ocho días antes del mismo, uso de dispositivos médicos al momento del aislamiento o $48 \mathrm{~h}$ antes (ventilación mecánica, catéter vesical, catéter vascular central), uso de antimicrobianos por más de 48 h en los últimos 30 días, uso de dos o más antimicrobianos por más de 48 h en los últimos 30 días, presencia de infección asociada al cuidado de la salud y tiempo de estancia hospitalaria previa al aislamiento de $P$. aeruginosa.

Para el análisis se utilizó el programa estadístico SPSS ${ }^{\circledR}$ versión 18.0 (SPSS Inc. Chicago, Il, USA). Para la descripción epidemiológica y clínica de la muestra estudiada se utilizó distribución de frecuencias. Para determinar los factores asociados con la probabilidad de adquirir infección por $P$. aeruginosa resistente, se hizo un análisis de casos y controles, evaluando previamente la presencia de interacción entre los posibles factores de riesgo. Para esto se hizo una regresión logística múltiple con aquellas variables independientes con una $p \leq 0,25$ en el análisis bivariado o que a criterio de los investigadores pudieran ser factores de riesgo de acuerdo a la literatura revisada. Los resultados son expresados como razón de disparidad (OR) con sus respectivos intervalos de confianza (IC) del 95\%.

\section{Resultados}

En total se evaluaron 140 pacientes, 70 en cada grupo. Las características clínicas, microbiológicas y epidemiológicas de los casos y los controles se presentan en la Tabla 1. El grupo de pacientes con aislamiento de 
P. aeruginosa MR (casos), tuvo como sistema más frecuentemente comprometido el urinario, estuvo expuesto en una mayor proporción a tratamiento antibacteriano con carbapenémicos y aminoglucósidos por más de $48 \mathrm{~h}$ en los últimos 30 días, recibió en mayor proporción dos o más antibacterianos en el mes previo y tuvo una mayor estancia hospitalaria previa al aislamiento.
En el análisis bivariado los factores asociados al desarrollo de infección por $P$. aeruginosa MR que alcanzaron significancia estadística fueron: el uso previo de carbapenémicos (OR 3,12- IC 1,21-8,03, p: 0,02), aminoglucósidos (OR 5,09- IC: 1,38-18,77, p: 0,01) y el tiempo de estancia previo al aislamiento (OR 1,03- IC: 1,01-1,05, p: 0,01) (Tabla 2).

Tabla 1. Características clínicas, microbiológicas y epidemiológicas de pacientes con Pseudomonas aeruginosa sensible y multi-resistente

\begin{tabular}{|c|c|c|c|}
\hline Variable & $\begin{array}{l}\text { Pacientes con } P \text {. aeruginosa } \\
\text { sensible } \\
(n=70)\end{array}$ & $\begin{array}{l}\text { Pacientes con } P \text {. aeruginosa } \\
\text { multi-resistente } \\
(\mathrm{n}=70)\end{array}$ & p \\
\hline Edad en años, media (DE) & $43,11(25,69)$ & $43,54(24,08)$ & 0,06 \\
\hline Sexo, mujer (\%) & $16(23)$ & $26(37)$ & 0,85 \\
\hline \multicolumn{4}{|l|}{ Co-morbilidades (\%) } \\
\hline Enfermedad pulmonar crónica & $2(3)$ & 4 (6) & 0,40 \\
\hline Enfermedad renal crónica & $9(13)$ & $11(16)$ & 0,63 \\
\hline Diabetes mellitus & $9(13)$ & $10(14)$ & 0,80 \\
\hline Trasplante de órgano solido & $0 \quad(0)$ & $1(1)$ & 0,31 \\
\hline Neoplasia maligna & $7(10)$ & $8(11)$ & 0,78 \\
\hline $\mathrm{VIH} / \mathrm{SIDA}$ & $0 \quad(0)$ & $2(3)$ & 0,15 \\
\hline Inmunosupresión por ME durante la hospitalización (\%) & $3(4)$ & $3(4)$ & 1,00 \\
\hline Quemadura como causa de hospitalización (\%) & $5(7)$ & $7(10)$ & 0,55 \\
\hline Cirugía durante la hospitalización (\%) & $44(63)$ & $45(64)$ & 0,86 \\
\hline \multicolumn{4}{|l|}{ Muestra biológica donde se hizo el aislamiento (\%) } \\
\hline Sangre & $15(21)$ & $9(13)$ & 0,18 \\
\hline Orina & $1(1)$ & $21(30)$ & $<0,05$ \\
\hline Tejido & $17(24)$ & $13(19)$ & 0,41 \\
\hline Hueso & $12(17)$ & $8(11)$ & 0,33 \\
\hline Pus & $8(11)$ & $6(9)$ & 0,57 \\
\hline Líquido peritoneal & $8(11)$ & $6(9)$ & 0,57 \\
\hline Secreción respiratoria & $3(4)$ & $6(9)$ & 0,30 \\
\hline Lavado broncoalveolar & $3(4)$ & $2(3)$ & 0,65 \\
\hline Líquido pleural & $1(1)$ & $0 \quad(0)$ & 0,32 \\
\hline Punta de catéter & 1 (1) & $0 \quad(0)$ & 0,32 \\
\hline \multicolumn{4}{|c|}{ Uso de dispositivos médicos al momento del aislamiento o en las últimas $48 \mathrm{~h}$ (\%) } \\
\hline Catéter venoso central & $19(27)$ & $22(31)$ & 0,58 \\
\hline Sonda vesical & $26(37)$ & $26(37)$ & 1,00 \\
\hline Ventilación mecánica & $11(16)$ & $11(16)$ & 1,00 \\
\hline \multicolumn{4}{|c|}{ Uso de antimicrobiano por más de 48 h en los 30 días previos (\%) } \\
\hline Cualquier antimicrobiano & $37(53)$ & $41(59)$ & 0,49 \\
\hline Carbapenémico (MEM /IMP) & $7(10)$ & $18(26)$ & 0,01 \\
\hline Fluoroquinolonas & $2(3)$ & $3(4)$ & 0,65 \\
\hline Piperacilina/tazobactam & $15(21)$ & $16(23)$ & 0,84 \\
\hline Aminoglucósidos & $3(4)$ & $13(19)$ & 0,01 \\
\hline Uso de dos o más antimicrobianos & $10(14)$ & $24(34)$ & 0,01 \\
\hline \multicolumn{4}{|l|}{ Otras características } \\
\hline Infección asociada al cuidado de la salud & $48(69)$ & $52(74)$ & 0,45 \\
\hline Estancia en UCl al momento del aislamiento (\%) & $18(26)$ & $19(27)$ & 0,85 \\
\hline Estancia hospitalaria previa al aislamiento, medias (DE) & $10,84(13,14)$ & $22,23(27,53)$ & 0,01 \\
\hline
\end{tabular}


Tabla 2. Análisis bivariado de los factores de riesgo para infección por $P$. aeruginosa multi-resistente

\begin{tabular}{|c|c|c|c|c|c|}
\hline Variable & $\begin{array}{l}\text { P. aeruginosa } \\
\text { sensible } \\
(n=70)\end{array}$ & $\begin{array}{l}\text { P. aeruginosa } \\
\text { multi-resistente } \\
(\mathrm{n}=70)\end{array}$ & OR & IC $95 \%$ & p \\
\hline Sexo mujer & 16 & 26 & 1,99 & $0,95-4,18$ & 0,07 \\
\hline \multicolumn{6}{|l|}{ Co-morbilidades } \\
\hline Enfermedad pulmonar crónica & 2 & 4 & 2,06 & $0,36-11,63$ & 0,41 \\
\hline Enfermedad renal crónica & 9 & 11 & 1,26 & $0,49-3,27$ & 0,63 \\
\hline Diabetes mellitus & 9 & 10 & 1,13 & $0,43-2,97$ & 0,80 \\
\hline Neoplasia maligna & 7 & 8 & 1,16 & $0,40-3,40$ & 0,78 \\
\hline Quemadura como causa de hospitalización & 5 & 7 & 1,44 & $0,44-4,79$ & 0,55 \\
\hline Cirugía durante la hospitalización & 44 & 45 & 1,06 & $0,53-2,12$ & 0,86 \\
\hline \multicolumn{6}{|c|}{ Uso de antimicrobiano por más de 48 h en los 30 días previos } \\
\hline Cualquier antimicrobiano & 37 & 41 & 1,26 & $0,64-2,45$ & 0,49 \\
\hline Carbapenémico (MEM/IMP) & 7 & 18 & 3,12 & $1,21-8,03$ & 0,02 \\
\hline Fluoroquinolonas & 2 & 3 & 1,52 & $0,25-9,40$ & 0,65 \\
\hline Piperacilina/tazobactam & 15 & 16 & 1,09 & $0,49-2,41$ & 0,84 \\
\hline Aminoglucósidos & 3 & 13 & 5,09 & $1,38-18,77$ & 0,01 \\
\hline Uso de dos o más antimicrobianos & 10 & 24 & 3,13 & $1,36-7,19$ & 0,01 \\
\hline \multicolumn{6}{|c|}{ Uso de dispositivos médicos al momento del aislamiento o en las últimas $48 \mathrm{~h}$} \\
\hline Catéter venoso central & 19 & 22 & 1,23 & $0,59-2,55$ & 0,58 \\
\hline Sonda vesical & 26 & 26 & 1,00 & $0,51-1,98$ & 1,00 \\
\hline Ventilación mecánica & 11 & 11 & 1,00 & $0,40-2,48$ & 1,00 \\
\hline \multicolumn{6}{|l|}{ Otras características } \\
\hline Infección asociada al cuidado de la salud & 48 & 52 & 1,32 & $0,63-2,76$ & 0,45 \\
\hline Estancia en UCl al momento del aislamiento & 18 & 19 & 1,08 & $0,51-2,28$ & 0,85 \\
\hline Estancia hospitalaria previo aislamiento, media días (DE) & $10,84(13,14)$ & $22,23(27,53)$ & 1,03 & $1,01-1,05$ & 0,01 \\
\hline
\end{tabular}

Tabla 3. Análisis multivariado de los factores de riesgo para infección por P. aeruginosa multi-resistente

\begin{tabular}{|c|c|c|c|c|c|}
\hline Variable & Coeficiente & Error estándar & Test de Wald & OR & IC $95 \%$ \\
\hline Sexo femenino & 0,84 & 0,41 & 4,07 & 2,31 & $1,02-5,2$ \\
\hline Estancia hospitalaria previa al aislamiento & 0,03 & 0,01 & 7,04 & 1,03 & $1,01-1,05$ \\
\hline Aminoglucósido por más de 48 h en los 30 días previos & 1,13 & 0,46 & 6,08 & 3,09 & $1,26-7,58$ \\
\hline Uso de dos o más antimicrobianos por más de 48 h en los 30 días previos & 1,48 & 0,71 & 4,37 & 4,4 & $1,1-17,65$ \\
\hline
\end{tabular}

En el análisis multivariado se encontró significancia estadística con la estancia hospitalaria (OR 1,03- IC 1,01-1,05) y el uso de aminoglucósidos (OR 1,30-19,28) o de dos o más antibacterianos en los últimos 30 días (OR 3,09- IC: 1,26-7,58) (Tabla 3). En este análisis se encontró que el riesgo de desarrollar una infección por $P$. aeruginosa MR es de 3\% por cada día de estancia hospitalaria previo al aislamiento de la bacteria; sin embargo, no hubo asociación alguna con las co-morbilidades estudiadas, con el uso de dispositivos, con el sitio de adquisición de la infección, ni con la estancia en UCI al momento del aislamiento; así como tampoco hubo asociación con el uso de fluoroquinolonas, piperacilina/tazobactam ni carbapenémicos.

\section{Discusión}

A nivel global $P$. aeruginosa MR es causa importante de infecciones asociadas al cuidado de la salud. Al igual que lo descrito en otros estudios, el uso de antimicrobia- 
nos y la estancia hospitalaria prolongada son factores de riesgo para desarrollar procesos infecciosos por esta bacteria en la población estudiada ${ }^{21-24,29-31}$. Adicionalmente, encontramos que por cada día de estancia el riesgo calculado para adquirir una infección por este microorganismo es de $3 \%$, sin asociarse con mayor estancia hospitalaria después del aislamiento microbiológico, contrario a lo reportado por otros autores ${ }^{26}$.

En el presente estudio se evaluó el uso de varios grupos de antimicrobianos en el mes previo al aislamiento del microorganismo MR y sólo se encontró significancia estadística en el análisis bivariado, con el uso de carbapenémicos y aminoglucósidos; sin embargo, en el análisis multivariado, el uso de aminoglucósidos fue el único relacionado con el desarrollo de infección por $P$. aeruginosa MR, hallazgo descrito por otros autores ${ }^{25,26}$. Esto es debido probablemente a que estos antimicrobianos tienen un impacto en la microbiota normal de los pacientes, predisponiendo para la adquisición de nuevas cepas o aumentando la expresión de microorganismos resistentes ya albergados por los mismos, además hay que tener en cuenta que el uso de aminoglucosidos en microorganismos $\mathrm{MR}$, no se realiza en monoterapia y encontramos que el estar expuesto a dos o más antibacterianos sí favorece el desarrollo de infecciones por esta bacteria.

La asociación descrita entre el uso de antimicrobianos y la presencia de $P$. aeruginosa MR, refuerza la importancia que tiene la implementación de políticas hospitalarias encaminadas al control del uso de antimicrobianos, que buscan disminuir la incidencia de infecciones por este tipo de bacterias ${ }^{27,28}$. En Colombia no son muchos los estudios que hayan valorado objetivamente el riesgo de multi-resistencia en nuestros hospitales. Villegas y cols., encontraron que en un brote de $P$. aeruginosa MR en un centro oncológico, el uso de ventilación mecánica y el tiempo de estancia, fueron los factores de riesgo más importantes; en el análisis multivariado la exposición a antimicrobianos no alcanzó significancia estadística ${ }^{6}$.

La presencia de dispositivos invasores como catéter venoso central, tubo oro-traqueal y sondas urinarias, han sido una constante dentro de los factores de riesgo relacionados con el desarrollo de infecciones por bacterias MR, no sólo por $P$. aeruginosa, sino también por $K$. pneumoniae y Acinetobacter baumannii ${ }^{30,32}$. En nuestro estudio, la presencia de estos dispositivos invasores no se asoció con mayor riesgo de multi-resistencia; esto puede ser explicado por las políticas adoptadas por nuestra institución para el adecuado manejo y cuidado de estos dispositivos.

Este estudio tiene varias limitaciones. El tamaño de la muestra posiblemente le da bajo poder para detectar diferencias entre los dos grupos respecto al uso previo de carbapenémicos, como se ha reportado en otros estu$\operatorname{dios}^{26,30}$. El diseño se limita a un estudio epidemiológico, donde los mecanismos de resistencia y la clonalidad no fueron analizados, pudiendo ser que los factores de riesgo para cada uno de ellos fueran diferentes; sin embargo, en la literatura la consideración de multi-resistencia por su expresión fenotípica (in vitro) es comúnmente usada independientemente al mecanismo de resistencia ${ }^{27,31-33}$.

Otro aspecto a tener en cuenta es que existen múltiples definiciones de MR descritas y algunos consensos, habiendo adoptado para este estudio la definición de MR como la falta de sensibilidad a dos o más familias de antibacterianos o a carbapenémicos ${ }^{33-37}$; esto posiblemente influyó en no encontrar asociación entre la MR y otros factores de riesgo que han sido descritos en estudios donde la MR es considerada como resistencia a tres o más familias de antibacterianos.

En conclusión, en la población estudiada, el desarrollar infección por $P$. aeruginosa MR está directamente relacionado con el uso previo de antimicrobianos, principalmente aminoglucósidos, y con el tiempo de estancia hospitalaria previo a la infección, lo cual es congruente con lo descrito en la literatura científica.

\section{Resumen}

Introducción: Pseudomonas aeruginosa multi-resistente (MR) es causa frecuente de infecciones asociadas al cuidado de la salud, su epidemiología es compleja y hay pocos estudios que permiten comprenderla. El estudio de los factores de riesgo asociados a este tipo de bacterias es necesario para la implementación de estrategias de control de las mismas. Objetivo: Determinar factores de riesgo asociados a infección por $P$. aeruginosa MR en pacientes hospitalizados en el Hospital Universitario de San Vicente Fundación-Medellín. Materiales y Métodos: Estudio analítico con abordaje como casos y controles para determinar factores de riesgo asociados a infección por $P$. aeruginosa MR. Resultados: Se incluyó un total de 140 pacientes, 70 en cada grupo. En el análisis bivariado se encontró asociación con el uso previo de carbapenémicos (OR 3,12- IC 1,21-8,03; p: 0,02), aminoglucósidos (OR 5,09- IC: 1,38-18,77; p: 0,01) y el tiempo por día de estancia previo al aislamiento (OR 1,03- IC: 1,01-1,05; p: 0,01). En el análisis multivariado hay asociación entre la estancia hospitalaria (OR 1,03- IC 1,01-1,05), el uso de aminoglucósidos (OR 1,30-19,28) y el uso de dos o más antimicrobianos en los últimos 30 días (OR 3,09- IC: 1,26-7,58) con el desarrollo de infecciones por $P$. aeruginosa $\mathrm{MR}$. El riesgo de desarrollar una infección por esta bacteria fue de $3 \%$ por cada día de estancia hospitalaria previo al aislamiento. Conclusión: El desarrollar infección por $P$. aeruginosa MR se asoció con el uso previo de antimicrobianos y con el tiempo previo de estancia hospitalaria. 


\section{Referencias bibliográficas}

1.- Giske C G, Monnet D L, Cars O, Carmeli Y. ReAct-Action on antibiotic resistance. Clinical and economic impact of common multidrugresistant gram-negative bacilli. Antimicrob Agents Chemother 2008; 52: 813-21.

2.- $\quad$ Tan T Y, Hsu L Y, Koh T H, Ng L S, Tee N W, Krishnan P, et al. Antibiotic resistance in gramnegative bacilli: a Singapore perspective. Ann Acad Med Singapore 2008; 37: 819-25.

3.- OMS. WHO Global strategy for containment of antimicrobial resistance, who/cds/csr/drs/2001/ en. [sede web]. Suiza: 2001. [Consultado el 12 de septiembre de 2012] Disponible en: http://www. who.int/csr/resources/publications/drugresist/ WHO_CDS_CSR_DRS_2001_2_EN/en/

4.- Rioua M, Carbonnellea S, Avraina L, Mesarosa N, Pirnayc J P, Bilocqc F, et al. In vivo development of antimicrobial resistance in Pseudomonas aeruginosa strains isolated from the lower respiratory tract of Intensive Care Unit patients with nosocomial pneumonia and receiving antipseudomonal therapy. Int $\mathrm{J}$ Antimicrob Ag 2010; 36: 513-22.

5.- Alliance for the Prudent Use of Antibiotics. Shadow epidemic the growing menace of drug resistance 2005. GAARD report. [sede web]. USA: 2005. [Consultado el 19 agosto de 2012] Disponible en: http://www.tufts.edu/med/apua/ about_us/publications.shtml

6.- Cortés J A, Cuervo S I, Urdaneta A M, Potdevin G, Arroyo P, Villegas M, et al. Identifying and controlling a multirresistant Pseudomonas aeruginosa outbreak in a LatinAmerican cancer centre and its associated risk factors. Braz J Infect Dis 2009; 13: 99-103.

7.- Sader H S, Jones R N, Gales A C, Silva J B, Pignatari A C, Grupo SENTRY. SENTRY Antimicrobial Surveillance Program Report: Latin American and Brazilian results for 1997 through 2001. Braz J Infect Dis 2004; 8: 25-79.

8.- Programa Europeo para la Vigilancia de Resistencia a Antimicrobianos. Reporte anual 2007 [sede web]. Holanda: EARSS; Octubre de 2008. [Consultado el 16 de agosto de 2012] Disponible en: http:/www.rivm.nl/earss/Images/ EARSS\%202007_FINAL_tcm61-55933.pdf.

9.- Grupo para el estudio de la resistencia a antibióticos de Medellín. Perfiles de sensibilidad de Pseudomonas aeruginosa [sede web]. Medellín: Germen; 2011 Disponible en: http:// www.grupogermen.org. [accedido el 19 de agosto de 2012].

10.- Pier G B, Ramphal R. Pseudomonas aeruginosa. En Mandell, Douglas \& Bennett's Principles and Practice of Infectious Diseases. Mandell G L, Bennett J E, Dolin R, eds. Capítulo 219. 7 ed. Philadelphia: Churchill Livingstone. An Imprint of Elsevier; 2010.

11.- Vila J, Marco F. Lectura interpretada del antibiograma de bacilos gramnegativos no fermentadores. Enferm Infecc Microbiol Clin 2002; 20 (6): 304-12.

12.- Ray G T, Baxter R, DeLorenze G N. Hospital-Level rates of fluoroquinolone use and the risk of hospital-acquired infection with ciprofloxacin-nonsusceptible Pseudomonas aeruginosa. Clin Infect Dis 2005; 41: 441-9.

13.- Maseda H, Sawada I, Saito K, Uchiyama H, Nacre T, Nomura N. Enhancement of the mexAB-oprMEfflux pump expression by a quorum-sensing autoinducer and its cancellation by a regulator, MexT, of the mexEF-opr $N$ efflux pump operon in Pseudomonas aeruginosa. Antimicrob Agents Chemother 2004; 48 (4): 1320-8.

14.- Cuzon G, Naas T, Villegas M V, Correa A, Quinn J P, Nordmann P. Wide dissemination of Pseudomonas aeruginosa producing beta-lactamase blaKPC-2 gene in Colombia. Antimicrob Agents Chemother 2011; 55: 5350-3.

15.- Villegas M V, Lolans K, del Rosario Olivera M, Suárez CJ, Correa A, Queenan A M, et al. Colombian Nosocomial Resistance Study Group. First detection of metallo-beta-lactamase VIM-2 in Pseudomonas aeruginosa isolates from Colombia. Antimicrob Agents Chemother 2006; 50: 226-9.

16.- Crespo M P, Woodford N, Sinclair A, Kaufmann M E, Turton J, Glover J, et al. Outbreak of carbapenem-resistant Pseudomonas aeruginosa producing VIM-8, a novel metallobeta-lactamase, in a tertiary care center in Cali, Colombia. J Clin Microbiol 2004; 42: 5094-101.

17.- Tzouvelekis L S, Markogiannakis A, Psichogiou M, Tassios P T, Daikos G L. Carbapenemases in Klebsiella pneumoniae and other Enterobacteriaceae: an evolving crisis of global dimensions. Clin Microbiol Rev 2012; 25: 682-707

18.- Jacoby T S, Kuchenbecker R S, Dos Santos R P, Magedanz L, Guzatto P, Moreira L B J. Impact of hospital-wide infection rate, invasive procedures use and antimicrobial consumption on bacterial resistance inside an intensive care unit. Hosp Infect 2010; 75: 23-7.

19.- Ikeda Y, Mamiya T, Nishiyama H, Narusawa S Koseki T, Mouri A, et al. A permission system for carbapenem use reduced incidence of drugresistant bacteria and cost of antimicrobials at a general hospital in Japan. Nagoya J Med Sci 2012; 74: 93-104.

20.- Chen Y C, Sheng W H, Wang J T, Chang S C, Lin H C, Tien K L, et al. Effectiveness and limitations of hand hygiene promotion on decreasing healthcare-associated infections. PLoS One 2011; 6: 27163.

21.- Kumar S H, De A S, Baveja S M, Gore M A. Prevalence and risk factors of metallo $\beta$-lactamase producing Pseudomonas aeruginosa and Acinetobacter species in burns and surgical wards in a tertiary care hospital. J Lab Physicians 2012; 4: 39-42.

22.- Rubio-Pérez I, Martin-Pérez E, García D D, Calvo M L, Barrera E L. Extended-spectrum beta-lactamase-producing bacteria in a tertiary care hospital in Madrid: epidemiology, risk factors and antimicrobial susceptibility patterns. Emerg Health Threats J 2012; 5. doi: 10.3402/ ehtj.v5i0.11589. Epub 2012 Jul 18.

23.- Vardi M, Kochavi T, Denekamp Y, Bitterman H. Risk factors for urinary tract infection caused by Enterobacteriaceae with extended-spectrum beta-lactamase resistance in patients admitted to internal medicine departments. Isr Med Assoc J 2012; 14: 115-8.

24.- Gedik H. Does antimicrobial use increase the rate of antimicrobial resistance? A one year experience. Indian J Med Microbiol 2012; 30: 198-202. doi: 10.4103/0255-0857.96692.

25.- Fortaleza C M, Freire M P, Filho D de C, de Carvalho Ramos M. Risk factors for recovery of imipenem- or ceftazidime-resistant Pseudomonas aeruginosa among patients admitted to a teaching hospital in Brazil. Infect Control Hosp Epidemiol 2006; 27: 901-6.

26.- Aloush V, Navon-Venezia S, Seigman-Igra Y, Cabili S, Carmeli Y. Multidrug-resistant Pseudomonas aeruginosa: risk factors and clinical impact. Antimicrob Agents Chemother. 2006; 50: 43-8.

27.- Charbonneau P, Parienti J J, Thibon P, Ramakers M, Daubin C, du Cheyron D, et al. Fluoroquinolone use and methicillin-resistant Staphylococcus aureus isolation rates in hospitalized patients: a quasi experimental study. Clin Infect Dis 2006; 42: 778-84.

28.- Livermore D M. Minimising antibiotic resistance. Lancet Infect Dis 2005; 5: 450-9.

29.- Paterson D L. Collateral damage from cephalosporin or quinolone antibiotic therapy. Clin Infect Dis 2004; 38: 341-5.

30.- Hussein K, Sprecher H, Mashiach T, Oren I, Kassis I, Finkelstein R. Carbapenem resistance among Klebsiella pneumoniae isolates: risk factors, molecular characteristics, and susceptibility patterns. Infect Control Hosp Epidemiol 2009; 30: 666-71.

31.- Tumbarello M, Repetto E, Trecarichi EM, Bernardini C, De Pascale G, Parisini A, et al. Multidrug-resistant Pseudomonas aeruginosa bloodstream infections: risk factors and mortality. Epidemiol Infect 2011; 139: 1740-9.

32.- Dent L L, Marshall D R, Pratap S, Hulette R B. Multidrug resistant Acinetobacter baumannii: a descriptive study in a city hospital. BMC Infect Dis 20107; 10: 196.

33.- Ortega B, Groeneveld A B, Schultsz C. Endemic multidrug-resistant Pseudomonas aeruginosa in critically ill patients. Infect Control Hosp Epidemiol 2004; 25: 825-31. 
34.- Magiorakos A P, Srinivasan A, Carey R B, Carmeli Y, Falagas M E, Giske C G, et al. Multidrug-resistant, extensively drug-resistant and pandrug-resistant bacteria: an international expert proposal for interim standard definitions for acquired resistance. Clin Microbiol Infect 2012; 18: 268-81.

35.- Ren C L, Konstan M W, Yegin A, Rasouliyan
L, Trzaskoma B, Morgan W J, et al. Multiple antibiotic-resistant Pseudomonas aeruginosa and lung function decline in patients with cystic fibrosis. J Cyst Fibros 2012; 11: 293-9.

36.- Erdem I, Kucukercan M, Ceran N. In vitro activity of combination therapy with cefepime, piperacillin-tazobactam, or meropenem with ciprofloxacin against multidrugresistant Pseudomonas aeruginosa strains. Chemotherapy 2003; 49: 294-7.

37.- Shanthi M, Sekar U. Multi-drug resistant Pseudomonas aeruginosa and Acinetobacter baumannii infections among hospitalized patients: risk factors and outcomes. J Assoc Physicians India 2009; 57: 638-40. 